\title{
Design and Application of an In Situ Illumination System for an Aberration- corrected Environmental Transmission Electron Microscope
}

\author{
Qianlang Liu ${ }^{1}$, Liuxian Zhang $^{1}$ and Peter A. Crozier ${ }^{2}$ \\ 1. School for the Engineering of Matter, Transport and Energy, Arizona State University, 85287, \\ Arizona, USA
}

Photocatalytic water splitting has been considered a promising technology for generation of clean, sustainable, and carbon-neutral fuels. Essentially, the photocatalytic materials enable the process of converting and storing the inexhaustible solar energy in the form of $\mathrm{H}_{2}$ molecules. It is now recognized that atomic level in situ observations are critical for understanding fundamental functionalities of catalytic materials. The active catalyst structures under reaction conditions are not necessarily the same as the initial structures. The detailed structure-reactivity relationships and deactivation behaviors of the catalysts can be developed by following the structural evolutions in situ. For photocatalysts, this requires that the system be observed in the presence of light, gas and thermal stimuli. There are several ways to introduce light illumination capability while observing the materials in an environmental transmission electron microscope (ETEM). Specimen holders that allow light illumination have been developed [1]. However, these designs do not allow heating/cooling of the catalysts. In this study, an optical fiber based in situ illumination system was designed and built for an aberration-corrected ETEM, FEI Titan. The ETEM was equipped with a monochromator and an aberration corrector providing sub-Angstrom image resolution. TEM hot stages can still be employed for in situ thermal processing of catalysts. This is critical for many fundamental studies on catalytic materials because thermal oxidizing or reducing treatments are often essential to create well-defined initial reference states of the materials. In this work, design considerations and applications of this illumination system will be discussed.

An in situ illumination system has been developed previously for an FEI Tecnai F20 ETEM [2]. Using this system, we have observed detailed structural evolution on $\mathrm{TiO}_{2}$ photocatalysts during exposure to in situ light and gas environments [3]. A similar design concept was applied here: an optical fiber guides the light into the ETEM and onto the TEM sample through the objective aperture port. With this design, the TEM specimen can be simultaneously exposed to light, different reactive gases, and heated up to $800^{\circ} \mathrm{C}$ using a Gatan heating holder. The geometry limitations, vacuum compatibility and performance optimization were taken into account in the design. As shown in Fig. 1, a fiber holder is needed to support the fragile optical fiber as well as maintaining the curvature $(25 \mathrm{~cm}$ in radius) needed to illuminate a tilted TEM specimen. The fiber optic (ordered from Ocean Optics) was custom made and a $30^{\circ}$ angle was cut at the end to refracted light onto a TEM sample (Fig. 2). The sample sits in a Gatan heating holder and needs to be tilted at $30^{\circ}$ in order to maximize the received light intensity. An LDLS EQ-99, laser driven xenon plasma source developed by Energetiq, is employed to provide illumination over the wavelength range $200-800 \mathrm{~nm}$. With such a light source, we are able to achieve close to 10 suns intensity across the entire wavelength range. It is essential to align the fiber with respect to the center of a TEM sample since the bright spot with highest intensity ( $>90 \%$ of the maximum intensity) is of size $400 \times 800 \mu \mathrm{m}$. A precise alignment procedure using a photomultiplier tube (PMT) was developed. In this method, a TEM sample with fluorescent particles is loaded into the microscope and upon irradiation by the electron beam, photons are emitted isotropically. A fraction of the photons travel through the fiber optic and reach the PMT connected at the end. Because light paths are reversible, optimum alignment can be achieved by adjusting the positions of the fiber to maximize the PMT signal. 
We are interested in applying this system to probe the in situ surface structural changes on $\mathrm{TiO}_{2}$ and $\mathrm{Ta}_{2} \mathrm{O}_{5}$ as they have shown interesting photocatalytic/photocorrosion behaviors. Model materials synthesized using hydrothermal methods will be used as they have well-defined morphologies. Fundamental study of surface crystal and electronic structural changes after exposing the catalysts to light and water vapor for extended periods of time will be conducted. Heterogeneity in the particle system may lead to different chemical changes on different surfaces. Probing this heterogeneity is also a very important goal. It is believed that with the capability of in-situ illumination and the superior resolution, deeper understanding of reaction and deactivation mechanisms of various photocatalyst systems will be gained.

\section{References:}

[1] F. Cavalca et al, Nanotechnology 23 (2012) 075705

[2] B.K. Miller, P.A. Crozier, Microsc. Microanal. 19 (2013) 461-469

[3] L. Zhang, B.K. Miller, P.A. Crozier, Nano Lett. 13 (2) (2013) 679-684

[4] The support from US Department of Energy (DE-SC0004954) and the help from Ben Miller and Karl Weiss from ASU, Joerg Jinschek and technicians from FEI, as well as the ASU machine shop are gratefully acknowledged.

(a)

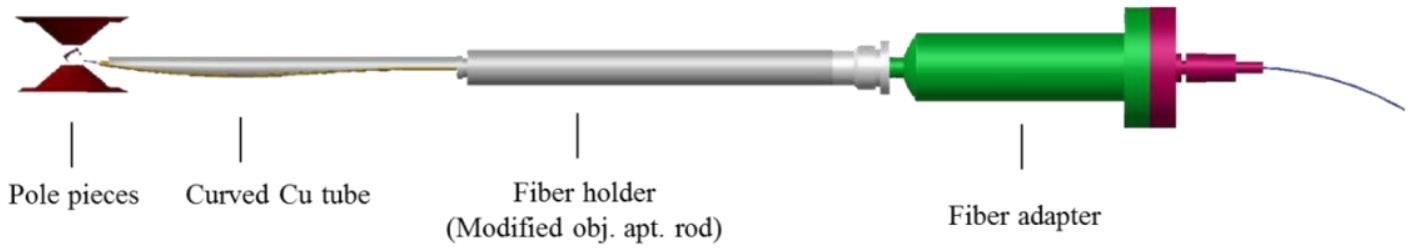

(b)
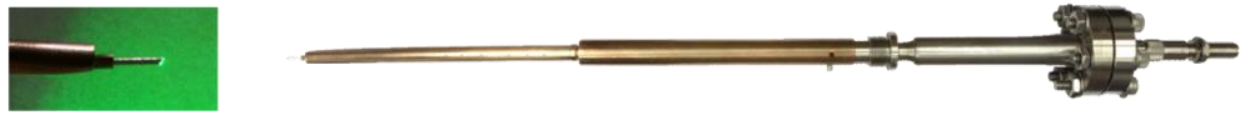

Figure 1: (a) Design drawing and (b) photographs of the fiber holder

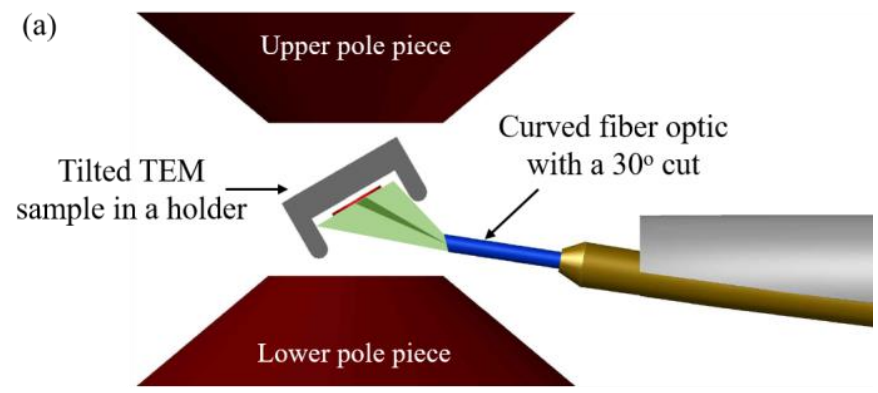

(b)

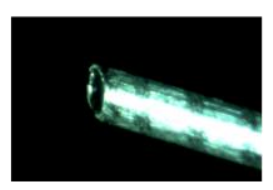

Figure 2: (a) Design drawing of the curved fiber illuminating a tilted TEM sample; (b) Photographs of the end of the fiber holder and the tip of the fiber. 